\title{
Mittelfristige Produktionsplanung bei ungewissen Szenarien ${ }^{1}$ )
}

\author{
Von D. Bartmann, München ${ }^{2}$ ) \\ Eingegangen am 15. März 1984
}

Zusammenfassung: In der mittelfristigen Produktionsplanung auf einem hochaggregierten Niveau spielt die Unsicherheit über die zukünftige Nachfrageentwicklung eine zentrale Rolle. Häufig werden deshalb Berechnungen unter verschiedenen Szenarien durchgeführt. Dies ist jedoch suboptimal. In der vorliegenden Arbeit werden Optimalitätsprinzipien formuliert, in denen die SzenariumEntwicklung bzw. die Nachfrageprognose durch einen stochastischen Prozeß dargestellt wird. Die Modelle sind vom Typ eines Markovschen Entscheidungsprozesses, d.h. sie erfüllen die Aktualisierung der Prognosen (Adaption), die vorausschauende Produktionsglättung (Antizipation) und die kosteneffiziente Absicherung gegen Fehleinschätzungen (Risiko) auf bestmögliche Weise.

Im ersten Teil der Arbeit werden für einige typische Situationen passende Optimierungsmodelle entwickelt, und zwar bei exogener Prognosevorgabe (Beispiel: Konzernmutter - Tochtergesellschaft), bei Produktionsplanung und Prognose in einer Hand, bei Prognose mit Selbstanpassung und bei Integration eines Frühwarnsystems. Es zeigt sich, daß die Trennung der Funktionen Planung und Prognose nachteilig sein kann.

Im zweiten Teil der Arbeit wird die entwickelte Theorie auf ein konkretes Beispiel aus der Automobilindustrie angewandt. Speziell wird die Frage untersucht: Wie steuert man die Produktion durch ein sich ankündigendes Absatztal bei Unsicherheit über Dauer und Stärke der Rezession? Anhand der Beispielrechnung wird deutlich gemacht, daß das stochastische Modell bei gleicher Kostenstruktur glattere Produktionskurven liefert als ein Modell mit festem Szenarium. Verantwortlich hierfür ist ein dem stochastischen Ansatz inne wohnender zusätzlicher kostenminimierender Trägheitseffekt.

Abstract: In medium term production planning at a highly aggregated level the uncertainty about future demand plays a central role. A widely used method to take the uncertainty into account is to investigate the same model with different scenarios. This approach produces only suboptimal results. In the first part of this paper some principles of optimality are formulated where forecasting is incorporated and future scenarios are treated as a stochastic process. The resulting models are of the type of a Markovian decision process. They have the property of actualization of forecasts (adaption), of looking ahead production smoothing (anticipation) and of efficient risk balancing. The different models are formulated in view of some typical situations occuring in practice. As a byproduct it is shown that the separation of long term forecasting and short term production planning may be disadvantageous.

The theory developed so far will then be applied to a concrete situation in the automotive industriy. In particular the problem investigated is how to control the production rate throughout

\footnotetext{
1) Auszug aus der Habilitationsschrift des Verfassers.

$\left.{ }^{2}\right)$ Dr. habil. Dieter Bartmann, Institut für Angewandte Mathematik und Statistik, TU München, Arcisstraße 21, D-8000 München 2.
} 
an imminent period of recession of unknown severity and duration. The computational results demonstrate that the model with a stochastic scenario yields smoother production lines than the model with a fixed scenario. This is due to an additional cost minimizing inertia caused by the stochastic law of motion.

\section{Einführung}

Selten hat eine Branche in letzter Zeit aufsehenerregendere Schlagzeilen gemacht als die Automobilindustrie. In der Automobilstadt Detroit gab es in den Wintermonaten $82 / 83$ zeitweise $25 \%$ Arbeitslose. Inzwischen haben die Automobilfirmen wieder Tritt gefaßt und bewegen sich in der Gewinnzone. Dieses Auf und Ab erhellt schlaglichtartig die zentrale Bedeutung der langfristigen Produktionsplanung. Sie steht im Spannungsfeld von Arbeitsmarktpolitik einerseits und Betriebsökonomik andererseits und erfordert aus dieser beiderseitigen Verpflichtung heraus ein Höchstmaß an Planungsqualität. Beträchtliche Planungsschwierigkeiten verursachen die Unsicherheiten über zukünftige Absatzzahlen. Die mittel- und langfristige Produktionsplanung stützt sich stets auf Nachfrageprognosen und ist deshalb unweigerlich mit dem Risiko einer Schieflage konfrontiert. Wie soll man dieses Risiko kalkulieren und was sind hinsichtlich der Ungewißheit über die zukünftige Entwicklung die optimalen Maßnahmen zur Lenkung des Prozesses?

Obwohl diese Frage für die Wirtschaftswissenschaft und Unternehmensforschung von zentraler Bedeutung ist, wurde sie erst in den füfziger Jahren von einer Reihe von Wirtschaftswissenschaftlern in das Zentrum ihrer Bemühungen gerückt [vgl. Streißler].

Um der Unsicherheit der Nachfrage Rechnung zu tragen, ist es gängige Praxis, die Modellberechinungen jeweils separat unter drei verschiedenen Szenarios (optimistisch realistisch, pessimistisch) durchzuführen. Von der Entscheidungstheorie her weiß man, daß dieses Vorgehen nicht optimal ist. Ein optimales Modell verlangt, daß man permanent sämtliche möglichen Szenarios im Auge behält bzw. während des gesamten Planungshorizonts Sprünge von einem Szenario in ein anderes zuläßt. Das bedeutet, daß man alle möglichen Entwicklungen auch in ihrer kostenmäßigen Konsequenz zu erfassen und bei der optimalen Entscheidungsfindung ins Kalkül zu ziehen hat. Ein Modell mit diesen geforderten Eigenschaften ist vom Typ eines stochastischen Sequentialentscheidungsprozesses.

Für die Anwendung eignen sich derartige Modelle bei stark abgegrenzten Problemstellungen, wie sie vornehmlich auf einer sehr tief liegenden Unternehmensebene auftreten, z.B. bei Problemen der Lagerhaltung [Hochstädter; Schneeweiß] der Produktionssteuerung bei Lebensmitteln mit geringer Haltbarkeit [Bartmann, 1983], oder der Kassenhaltung [Eppen \& Fama, Inderfurt].

In dieser Arbeit wird gezeigt, wie geeignete stochastische Modelle auch bei langfristigen Produktionsplanungsproblemen auf einer hohen Unternehmensebene zur Anwendung gelangen können.

Die typischen Fragestellungen beziehen sich auf die Nachfrageprognosen (Wie verläuft die erwartete Nachfrageentwicklung und wie sicher ist diese Erwartung?), auf 
die antizipierende Anpassung der Produktion (Produktionsrate und Produktionskapazität) an den Nachfrageprozeß (Soll man bei einem vorherzusehenden Absatztal Kapazität abbauen oder halten?) und auf $A$ bsicherung gegen Fehleinschätzung der Nachfragen (Lassen sich im Lager kritische Bestandszonen definieren, die ein Abweichen vom bisherigen Produktionsplanungskonzept erfordern?).

Der Planungshorizont erstreckt sich über einige Jahre. Dies fordert die optimale Kalkulation des Risikos und damit den Einsatz stochastischer Entscheidungsmodelle geradezu heraus.

Im ersten Teil der Arbeit wird der Frage nachgegangen: Wie formuliert man ein praxisgerechtes Modell? Im Mittelpunkt steht dabei die optimale Einbindung der Prognose in den Entscheidungsprozeß. Es werden für einige innerbetriebliche Situationen die entsprechenden Optimalitätsprinzipien entwickelt. Im zweiten Teil werden anhand eines Beispiels die Fragen behandelt, wie das Modell angewendet werden kann und welche Vorteile es gegenüber den deterministischen Ansätzen aufweist.

\section{Das stochastische Produktionsglättungsmodell von Beckmann}

Das Problem lautet:

Gegeben ist eine Nachfrageprognose sowie der augenblickliche Bestand in den Auslieferungslägern und beim Handel. Die Produktionsrate soll so gesteuert werden, daß man stets im günstigsten Stückkostenbereich produziert. Als Handlungsspielraum stehen zur Verfügung: Personaleinstellung/-sstop, vorgezogene Rente, Werksferien, Überstunden, zusätzliche Schichten und Marketingaktionen. Die Entscheidungen werden jeweils zu Beginn einer Planungsperiode (z.B. Quartal) getroffen.

Grundlegend für die Modellbildung ist das stationäre Produktionsglättungsmodell von Beckmann.

Sei

$u \quad$ Nachfrage im vorliegenden Quartal. $u$ ist zum Zeitpunkt der Entscheidung noch unbekannt und deshalb eine Zufallsvariable mit der Verteilungsfunktion $\psi(u)$.

$x \quad$ alte Produktionsrate

$y \quad$ alter Lagerbestand

$d \quad$ vorzunehmende Änderung der Produktionsrate

$\ddot{A K}$ Änderungskosten

$P K \quad$ Produktionskosten

$L$ erwartete Lager- und Fehlmengenkosten im vorliegenden Quartal

$N \quad$ Planungshorizont

$n \quad$ Anzahl der Quartale bis zum Planungshorizont

$\beta$ Diskontfaktor, $0<\beta<1$

$v_{n} \quad$ zu minimierende erwartete Gesamtkosten bei noch $n$ Quartalen bis zum Planungshorizont 
Das Optimierungsmodell ist rekursiv über $v_{n}$ definiert. $v_{n}$ hängt vom augenblicklichen Zustand des Systems ab. Dieser wird beschrieben durch die Zustandsvariablen $x$ und $y$. Die Entscheidungsvariable ist $d$. Sie ist so zu wählen, daß die neue Produktionsrate $x+d$ auch hinsichtlich aller zukünftigen Nachfrageprognosen günstig liegt. Die Optimierung von $d$ geschieht nach dem folgenden Prinzip der Optimalität

$$
\begin{aligned}
v_{n}(x, y)= & \min _{d}\{P K(x, d)+\ddot{A} K(x, d)+L(x, d, y)+ \\
& \left.+\beta \int v_{n-1}(x+d, y+x+d-u) d \psi(u)\right\} \quad n=1,2,3, \ldots
\end{aligned}
$$

und $v_{0}=V$ (Terminalauszahlung).

Die Terminalauszahlung $V$ wird bei stationären Problemen mit unendlichem Planungshorizont o.B.d.A. identisch null gesetzt. Sie beeinflußt die optimale Entscheidung nicht, da ihr Gegenwartswert verschwindend gering ist.

Die Lösung der obigen Funktionalgleichung (1) liefert eine Entscheidungsregel $\delta$ für alle möglichen Situationen zum jeweiligen Quartalsbeginn:

$$
\delta: \text { falls Zustand }(x, y) \text { beobachtet } \Rightarrow \text { wähle Aktion } d(x, y) \text {. }
$$

$\delta$ ist für alle Entscheidungszeitpunkte gleich. Dies ist bereits ein erster Anlaß zu kritischen Bemerkungen. Auf der einen Seite formuliert man ein kompliziertes stochastisches Modell, um dem Eventualcharakter zukünftiger Ereignisse Rechnung zu tragen, und auf der anderen Seite verschließt man die Augen vor exogenen Einflußfaktoren, die zumindest und in der einfachsten Form als zeitabhängige Größen auftreten. Bei der Berücksichtigung zeitabhängiger Größen wird man unwillkürlich mit Prognoseproblemen konfrontiert.

\section{Erweiterung auf Modelle mit Prognose}

In praxi ist die Prognosebildung ein unverzichtbarer Bestandteil der Produktionsplanung. Die Prognosen sind auch für das vorliegende Modell ein geeignetes Mittel zur Kopplung der endogenen Modellabläufe an exogene Prozesse. Es wird deshalb das Grundmodell (1) um den Begriff ,Prognose“ erweitert. Aus dem weiten Feld der Prognosetechniken sind im Zusammenhang mit sequentiellen Entscheidungsprozessen jedoch nur solche Verfahren von Belang, die sich in die rekursive Definition des Entscheidungsprozesses einbinden lassen. Es werden einige Möglichkeiten aufgeführt. Da es sich in der vorliegenden Arbeit um Mittel- und Langfristprobleme handelt, bleiben autoregressive Prognosemechanismen (z.B. ARIMA - Methoden oder autoadaptive Clättungsmethoden) außer Betracht [siehe hierzu Bartmann, 1979, 1984]. 


\subsection{Exogene Prognosevorgabe}

Es wird angenommen, daß die Produktionsplanung von der Prognoseerstellung getrennt ist. Auf diese Situation stößt man z.B. bei Konzernen.

Konzernmutter (KM) — Tochtergesellschaft macht erstellt Szenario- Produktionsplanung (PPL) prognose $z$

(KM) besitze ein ökonometrisches Modell, mit dem der Marktverlauf als Funktion einiger Wirkungsfaktoren $\left(e_{1}, e_{2}, \ldots, e_{L}\right)$ erklärt wird. Daraus wird ein Szenario $\left(e_{1}, e_{2}, \ldots, e_{L}\right) \rightarrow z$ gebildet und als Quartalsprognose an (PPL) weitergegeben.

Der Abteilung (PPL) bei der Tochtergesellschaft ist der Prognosemechanismus verborgen. Um zukünftige Nachfrageschwankungen antizipieren zu können, muß die optimale Produktionsanpassung $d^{*}$ das System in einen Zustand führen, der idealer Ausgangszustand nicht nur hinsichtlich des nächsten, sondern auch der folgenden Quartale ist. Dazu ist es erforderlich, auch die Nachfragen in der weiteren Zukunft abzuschätzen. Aufgrund der rekursiven Form des Entscheidungsprozesses (1) genügt es, die neue Konzernprognose zu kennen. Sie ist jedoch bei (PPL) unbekannt. Deshalb muß (PPL) Mutmaßungen darüber anstellen, wie die neue Konzernprognose ausfallen wird. Sie erreicht das, indem sie sie als Zufallsvariable auffaßt und dafür eine Verteilungsfunktion festlegt. Das Grundmodell (1) muß deshalb erweitert werden.

Es bezeichnet

$z$ aktuelle Konzernprognose (für das vorliegende Quartal)

$u \quad$ aus der Sicht von (PPL) die durch $z$ bedingte Quartalsnachfrage mit der Verteilungsfunktion $\psi(u \mid z)$

$w \quad$ nächste Konzemprognose (für das folgende Quartal), aus der Sicht von (PPL); Zufallsvariable mit der Verteilungsfunktion $\phi(w)$.

Das Optimierungsmodell ist jetzt definiert über das Dynamische Programm

$$
\begin{aligned}
& v_{n}(x, y, z)=\min _{d}\{P K(x, d)+\ddot{A} K(x, d)+L(x, d, y, z)+ \\
& +\beta \iint v_{n-1}(x+d, y+x+d-u, w) d \psi(u\lceil z) d \phi(w)\} \\
& \quad n=1,2,3, \ldots
\end{aligned}
$$

und $v_{0}=V$.

Der Vorteil von Modell (2) gegenüber (1) und damit der Wert der Prognose wird dadurch begründet, daß sich jetzt vermöge $z$ die Verteilung der Nachfrage stärker um ihren Erwartungswert konzentrieren läßt (falls die Prognosen gut sind!).

Dieser Gewinn geht jedoch zumindest teilweise wieder verloren, da bezüglich $w$ neue Unsicherheit ins Modell getragen wird. Das drückt sich im Doppelintegral in (2) 
aus. Die äußere Integration glättet die Kostenunterschiede zwischen günstigen und ungünstigen Zuständen (die Kostenkurve $v$ wird flacher!).

So zeigt sich hier deutlich, daß sich die Trennung von Prognose- und Planungsfunktion auf zwei verschiedene eigenverantwortliche Bereiche nachteilig auswirkt, sobald man dem Bereich (PPL) die Langfristprognose vorenthält.

Anders wäre es, wenn (PPL) bereits $w$ kennen würde. Dann entfiele die äußere Integration. Nun ist es aber häufig der Fall, daß bei (KM) $w$ schon relativ genau bekannt ist. Dies liegt in der Natur der Konzernprognosen, da sie sich meist auf globale Faktoren abstützen, die ihrerseits träge sind. Das soll im folgenden Modell ausgenützt werden.

\subsection{Produktionsplanung und Prognose in einer Hand}

Wir nehmen jetzt an, daß die Prognosen im eigenen Haus durchgeführt werden und die Abteilungen Prognose und Produktionsplanung ohne Zeitverlust miteinander kommunizieren. Dann läßt sich das Wissen um die Langfristprognose in das Entscheidungsmodell einbauen. Falls zu jedem Entscheidungszeitpunkt $t$ neben $z_{t}$ bereits die neue Prognose $w$ bekannt ist und $w$ auch nachträglich nicht mehr korrigiert wird, dann bedeutet dies, daß von Anfang an die Gesamtmarktprognosen $z_{1}, z_{2}, \ldots, z_{T}$ von sämtlichen Quartalen des Planungshorizonts $T$ festgelegt sind. Dann wird aus der bedingten Verteilung $\psi\left(u_{t} i z_{t}\right)$ die Verteilung $\psi_{t}\left(u_{t}\right)$. Es sei $v_{t}$ der Vektor der zu erwartenden Gesamtkosten bis zum Ende des Planungshorizonts $T$ bei optimaler Steuerung. Das Prinzip der Optimalität lautet

$$
\begin{array}{r}
v_{t}\left(x_{t}, y_{t}\right)=\min _{d_{t}}\left\{P K\left(x_{t}, d_{t}\right)+\ddot{A} K\left(x_{t}, d_{t}\right)+L\left(x_{t}, d_{t}, y_{t}\right)+\right. \\
\left.+\beta \int v_{t+1}\left(x_{t}+d_{t}, y_{t}+x_{t}+d_{t}-u_{t}\right) d \psi_{t}\left(u_{t}\right)\right\} \\
t=1,2, \ldots, T
\end{array}
$$

und $v_{T+1}=V$.

\subsection{Prognose mit Selbstanpassung}

Wir wählen nun ein Mittelding zwischen den beiden vorangegangenen extremen Alternativen " $w$ ist Zufallsvariable mit stationärer Verteilung" und " $w$ ist für alle Perioden bekannt" und formulieren $z_{t+1}$ als Zufallsvariable mit einer Verteilung, die von $z_{t}$ und der letzten Beobachtung $u_{t}$ abhängt. Die Verteilungsfunktion sei $\phi_{t}\left(z_{t+1} \mid z_{t}, u_{t}\right)$. Mit $z_{t}$ und $u_{t}$ läßt sich ein Maß für die Prognosequalität bilden. Man kann $\phi\left(z_{t+1} \mid z_{t}, u_{t}\right)$ so festlegen, daß einem großen Prognosefehler in geeigneter Weise Rechnung getragen wird. Nach dem Optimalitätsprinzip gilt 


$$
\begin{aligned}
\nu_{t}\left(x_{t}, y_{t}, z_{t}\right)= & \min _{d_{t}}\left\{P K\left(x_{t}, d_{t}\right)+\ddot{A} K\left(x_{t}, d_{t}\right)+L\left(x_{t}, d_{t}, y_{t}, z_{t}\right)+\right. \\
& +\beta \iint v_{t+1}\left(x_{t}+d_{t}, y_{t}+x_{t}+d_{t}-\right. \\
& \left.\left.-u_{t}, z_{t+1}\right) d \psi_{t}\left(u_{t} \mid z_{t}\right) \cdot d \phi_{t}\left(z_{t+1} \mid z_{t}, u_{t}\right)\right\} \quad t=1,2, \ldots, T
\end{aligned}
$$

und $v_{T+1}=V$.

Auf diese Weise ist ein Adaptionsmechanismus ins Modell aufgenommen.

\subsection{Integration eines Frühwarnsystems}

Die Idee der Selbstanpassung läßt sich noch enger an den praktischen Gegebenheiten formulieren. Vergegenwärtigen wir uns wieder die Situation Konzernmutter - Tochtergesellschaft. (KM) beobachtet die globalen Entwicklungen. Die Tochtergesellschaft hat den Kontakt zum Kunden. Sie kann deshalb Informationsquellen ausschöpfen, die (KM) verschlossen sind. (PPL) beobachtet die kurzfristig sich auswirkenden Marktanteilskämpfe (Werbung, Preis, Modellpflege, .. .). Sie zeigen sich als Abweichungen der beobachteten Nachfrage $u^{t}=\left(u_{t}, u_{t-1}, \ldots\right)$ bis zur Zeit $t$ von derjenigen Nachfragekurve, die sich aus dem von der Konzemprognose abgeleiteten Marktanteil ergeben müßte. Wir aggregieren diese Marktanteilsverschiebung evtl. zusammen mit weiterer Information zu einem Indikator $\eta$.

$\eta$ Frühwarnsignal, aktuelle Beobachtung

$\rho$ Frühwarnsignal zu Beginn des nächsten Quartals, Zufallsvariable mit der Verteilungsfunktion $\Theta(\rho)$.

Jetzt wird $\eta$ eine zusätzliche Zustandsvariable. Sie beeinflußt die Verteilung von $z_{t+1}$. Die bedingte Verteilungsfunktion von $z_{t+1}$ ist $\phi\left(z_{t+1} \mid z_{t}, u_{t}, \eta\right)$. Das Prinzip der Optimalität lautet

$$
\begin{gathered}
v_{t}\left(x_{t}, y_{t}, z_{t}, \eta\right)=\min _{d_{t}}\left\{P K\left(x_{t}, d_{t}\right)+\ddot{A} K\left(x_{t}, d_{t}\right)+L\left(x_{t}, d_{t}, y_{t}, z_{t}\right)+\right. \\
+\beta \iiint v_{t+1}\left(x_{t}+d_{t}, y_{t}+d_{t}-u_{t}, z_{t+1}, \rho\right) \\
\left.+d \psi\left(u_{t} \mid z_{t}\right) d \phi_{t}\left(z_{t+1} \mid z_{t}, u_{t}, \eta\right) d \Theta(\rho)\right\} \\
t=1,2, \ldots, T
\end{gathered}
$$

und $v_{T+1}=V$. 


\section{Beispiel: Automobilabsatz in der Ölkrise}

Obwohl auf sehr hoher Ebene formuliert, sind die oben entwickelten Modelle keineswegs Globalmodelle.

Änderungen der Produktionszahlen verlangen, sofern sie wesentlich sind, beträchtliche Aktivitäten und wirken ihrerseits in weite Bereiche eines Unternehmens hinein. Aus der globalen Sicht der Unternehmensleitung stellt sich die Frage nach der langfristigen Festlegung der Kapazitätsgrenzen und deren schrittweiser Ansteuerung. Dieses Problem besitzt einen hohen Komplexitätsgrad. Die Erfahrung hat jedoch gezeigt, daß zufriedenstellende Aussagen nicht mit Hilfe eines gigantischen Gesamtmodells zu erzielen sind. Vielmehr sollte man, nachdem die Gesamtproblematik in hochaggregierter Form herausgearbeitet wurde, weitgehend autonome Teilaspekte formulieren und in nachgeordnete Problemstellungen aufgliedern. Die einzelnen Teilprobleme werden dann mit Hilfe adäquater Methoden untersucht. Der Entwicklung der Modelle nach diesem Top-Down-Prinzip folgt das Zusammentragen und Verdichten der Ergebnisse von unten nach oben, so daß man im letzten Schritt für das Problem auf der obersten Hierarchiestufe Vorschläge zur Entscheidungsfindung erarbeitet.

Es ist für die Anwendung sehr wichtig, sich über die Stellung des stochastischen Produktionsglättungsmodells innerhalb des Gesamtproblemfeldes klar zu werden. Das Produktionsglättungsproblem besitzt sowohl einen operationalen als auch einen strategischen Aspekt. Das läßt sich sehr gut an dem im folgenden präsentierten Beispiel aus der Automobilindustrie beobachten. Das Beispiel wurde fur die Veröffentlichung vereinfacht.

Die erste Ölkrise verursachte einen gewaltigen Nachfrageeinbruch nach PKWs. Es war 1973 nicht genau abzusehen, wie stark dieser Einbruch sein würde, wie lange er anhalten würde und auf welches Niveau sich die Nachfrage langfristig wieder einpegeln würde. Für die Demonstration des stochastischen Modells ist diese Situation vortrefflich geeignet.

Wir versetzen uns deshalb zurück ins Jahr 1973. Für eine Szenarioprognose stehen die Nachfragezahlen von 1965 bis Mitte 1973 zur Verfügung. Es wird nur der Inlandsmarkt betrachtet.

\subsection{Prognose der Nachfrage}

Sei $B(t)$ die PKW-Dichte und $e(t)$ die Bevölkerung über 18 Jahre, dann ist die Prognose des Gesamtbestandes (Szenario)

$$
z(t)=B(t) \cdot e(t)
$$

$B(t)$ wird mit Hilfe eines ökonometrischen Modells geschätzt. Aus (6) läßt sich die jährliche Gesamtnachfrage $u_{G}$ bestimmen. Sie setzt sich zusammen aus dem Marktwachstum und dem Ersatz stillgelegter Fahrzeuge. Wir unterstellen eine mittlere Lebensdauer eines Autos von acht Jahren (Technologie der siebziger Jahre!). Es ist

$$
u_{G}(t)=\dot{B} e(t)+B(t-8) e(t-8)
$$


Die Prognose von $u_{G}$ läßt erkennen, daß sich der Nachfrageeinbruch bis 1977 auswirken wird. Es ist zu erwarten, daß er sich acht Jahre später in abgeschwächter Form wiederholt, weil dann die Ersatznachfrage entsprechend niedrig ist. Das bedeutet einen erneuten Nachfrageeinbruch im Zeitraum 1981 bis 1983. Siehe hierzu Abbildung 1.

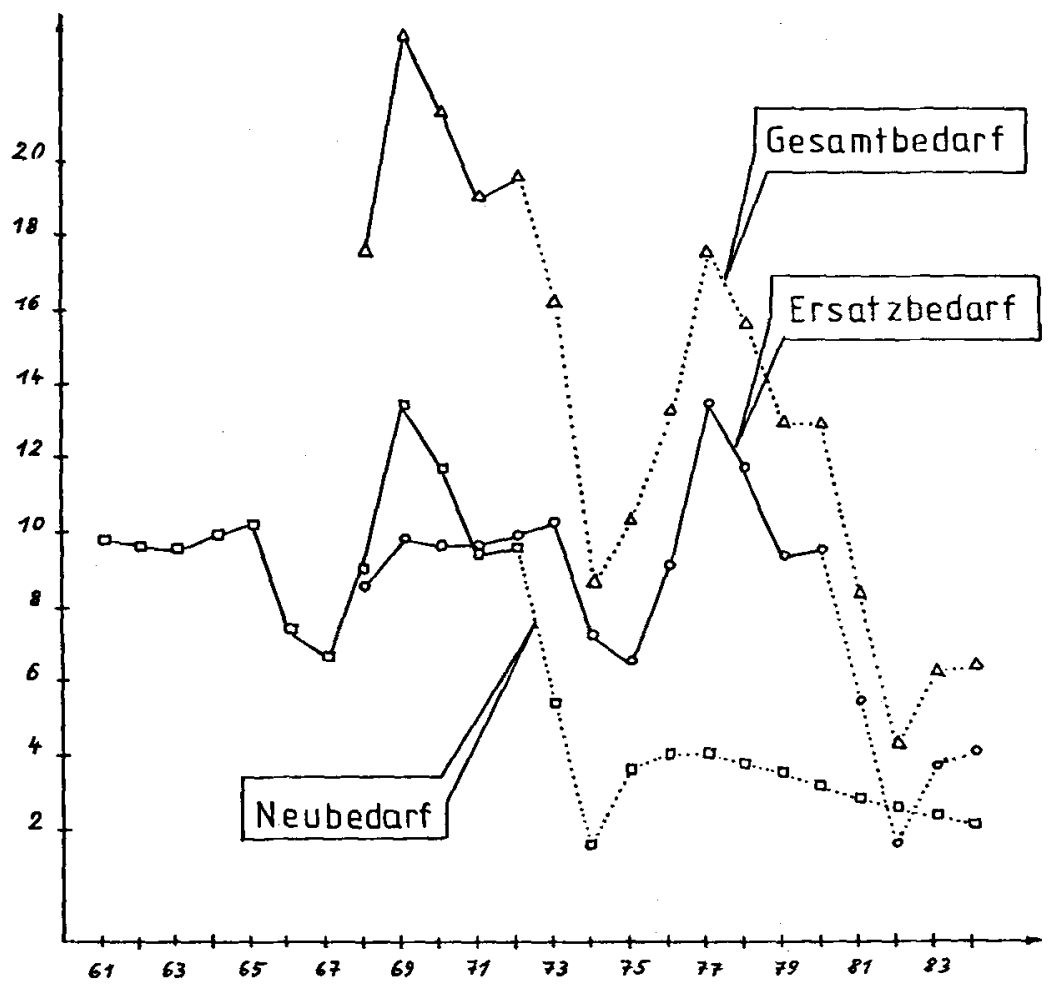

Abb. 1: Gesamtnachfrage $u_{G}$

Kennt man den eigenen Martkanteil $M(t)$, läßt sich aus der Gesamtnachfrage sofort der Prognosepfad der eigenen Nachfrage ableiten

$$
\hat{u}(t)=u_{G}(t) \cdot M(t) .
$$

Das Unternehmen besitze im Augenblick einen Marktanteil von 50\% und sei damit der größte Anbieter. Der daraus resultierende Vorsprung in der Produktionserfahrung wird dazu führen, daß sich der eigene Marktanteil in den nächsten zehn Jahren auf $60 \%$ erhöht und dann konstant bleibt. Demgemäß erhält man

\begin{tabular}{|l|l|l|l|l|l|l|l|l|l|l|l|l|}
\hline Jahr & 73 & 74 & 75 & 76 & 77 & 78 & 79 & 80 & 81 & 82 & 83 & 84 \\
\hline PKW & 32 & 18 & 22 & 28 & 39 & 35 & 30 & 30 & 20 & 10 & 15 & 15 \\
\hline
\end{tabular}

Tab. 1: Prognosepfad $\hat{u}(t)$ der Nachfrage (Einheit: 25000 PKW) 
Diese Zahlen machen deutlich, daß das Problem sowohl einen operationalen als auch einen strategischen Aspekt besitzt. Der operationale Aspekt trifft den kurzfristigen Bereich und befaßt sich mit der Frage, die vorliegende Situation optimal zu meistern, d.h. die sich ankündigende Talsohle optimal zu durchsteuern. Der strategische Aspekt wird deutlich, wenn man beachtet, daß die Wachstumsfunktion der PKW-Dichte 1973 ihren Wendepunkt längst hinter sich hat und sich die Wachstumsrate ständig verringert. Deshalb weist die Nachfragekurve auch in der Tendenz nach unten. Es ist zu überlegen, wie man diesem Effekt entgegenwirken kann. Das geschieht jedoch außerhalb des Modells (vom heutigen Standpunkt aus weiß man, daß dies möglich war). Innerhalb des Modells wird aber bereits berücksichtigt, daß die Langfristentwicklung durchaus anders verlaufen kann. Die Ungewißheit des Szenarios induziere einen stochastischen Nachfrageprozeß

$$
u(t)=\hat{u}(t)+\epsilon_{t}
$$

$\epsilon_{t}$ ist unabhängig und identisch normalverteilter Zufallsvariable mit Verteilungsfunktion $P\left(\epsilon_{t}\right)=N(0,3)$.

\subsection{Kostenstruktur}

\section{Lagerkosten}

Es sind neben dem Deckungsbeitrag der Lagerkosten die Kapitalbindungskosten anzusetzen. Beides sind proportionale Kosten im Vertriebslager.

Daneben entstehen auch noch Kosten in den Handelslägern. Dem Handel sind als Serviceleistung Idealbestände in seinen Lägern mitgeteilt. Er wird seine Bestellpoltik danach ausrichten. Es besteht aber die Möglichkeit, Waren in den Handel ,hineinzudrücken". Hier stellt sich die Frage nach der Definition der Idealbestände. Sollen sie ideal sein im Sinne eines besten Kompromisses zwischen Liefererfullungsgrad und Kapitalbindungskosten auf Seiten des Händlers oder ideal im Sinne des Gesamtsystems Produktion - Vertrieb?

Selbstverständlich kann der Handel nicht alleine den Preis in Form erhöhter Kapitalbindung dafür bezahlen, daß die Produktion auf einer kostengünstigeren Linie gefahren werden kann. Regulationen sind denkbar, z.B. über Zahlungsziel oder Skonti. Diese Kosten werden ebenfalls den Lagerkosten zugeschlagen.

\section{Fehlmengenkosten}

Fehlmengen führen nicht zum Umsatzverlust. Die Autos werden nachgeliefert (Lieferfristen). Als Fehlmengenkosten entstehen die Zwischenfinanzierungskosten des erst später realisierten Gewinnes. Da diese Kosten in der Regel geringer sind als die Lagerkosten, würde ein optimales Programm hohe Fehlbestände ansteuern. Das würde zu sehr langen Lieferfristen führen. 
In bestimmten Grenzen sind Lieferfristen zwar beim Kunden erwünscht, weil er damit ein hohes Image des Produktes verbindet, jedoch überwiegt bei längeren Wartezeiten die Ungeduld. Sie wird in Form von Strafkosten im Modell ausgedrückt. Sie sind abhängig von der Höhe der Fehlbestände und von der Marktposition des Unternehmens.

\section{Material- und Energiekosten}

Die Energie- und Materialkosten einschließlich des Umlaufvermögens in den Fertigungslägern werden als proportionale Kosten veranschlagt. Sie bieten kaum einen Spielraum zur Optimierung.

\section{Nichtmaterialkosten}

In ihnen sind sowohl die Lohnkosten in der Fertigung als auch die Kosten des gesamten Overheads mit Ausnahme des Lagers enthalten. Als Stückkosten hängen sie stark von der Produktionsrate ab.

Die Lohnkosten in der Fertigung sind in der ersten Näherung proportional zum Ausstoß. Es ist jedoch zu berücksichtigen, daß die Fertigung im wesentlichen in vollen Schichten gefahren wird. Ist eine Schicht nicht voll ausgelastet, entstehen zusätzliche Leerlaufkosten. Das sind Kosten für die Kapazitätsvorhaltung. Sie treten sprunghaft beim Übergang zur nächsten Schicht auf und fallen zur vollen Schicht hin linear bis null ab. Die Kostenkurve gliedert sich deshalb in drei Bereiche gemäß der Produktion im Einschicht-, Zweischicht- oder Dreischich tbetrieb.

Es ist noch eine weitere Modfikation notwendig. In den Bereichen hoher Überkapazität kann man den Entlassungen durch Kurzarbeit vorbeugen. Dadurch verringern sich die Leerlaufkosten. Jeder Schichtenbereich wird also wieder in zwei Teile unterteilt. Im jeweiligen linken Teil wird Kurzarbeit geleistet. Die Lohnkostenkurve in der Fertigung setzt sich deshalb aus insgesamt sechs geraden Stücken über den sechs Bereichen $B_{1}$ bis $B_{6}$ zusammen (siehe Abbildung 2).

Die restlichen Nichtmaterialkosten mit Ausnahme der Lagerkosten und der Kosten für das Ändern der Produktionsrate werden im sog. Overhead subsummiert. Diese Kostenkurve ist vom selben Typ wie die Lohnkostenkurve, jedoch mit kleinerer Steigung.

Die Summe aus Lohn- und Overheadkosten führt somit zu einer stückweise linearen Nichtmaterialkosten-Kurve wie in Abbildung 2.

Sei $\left[x^{-} ; x^{+}\right]$der zulässige Spielraum für die Produktionsrate $x$. Dann zerlegen die fünf Trennpunkte $T P_{1}, \ldots, T P_{5}$ das Gesamtintervall des Produktionsbereiches in sechs Einzelbereiche $B_{1}, \ldots, B_{6}$. Setzt man $x^{-}$für $T P_{0}$ und $x^{+}=T P_{6}$, so ist $B_{i}=\left[T P_{i-1} ; T P_{i}\right), i=1,2, \ldots, 5$ und $B_{6}=\left[T P_{5} ; x^{+}\right] . F K_{t, i}$ ist der Fixkostenanteil und $P K_{t, i}$ ist der proportionale Kostensatz der Nichtmaterialkosten im Bereich $B_{i}$ zur Periode $t$. 


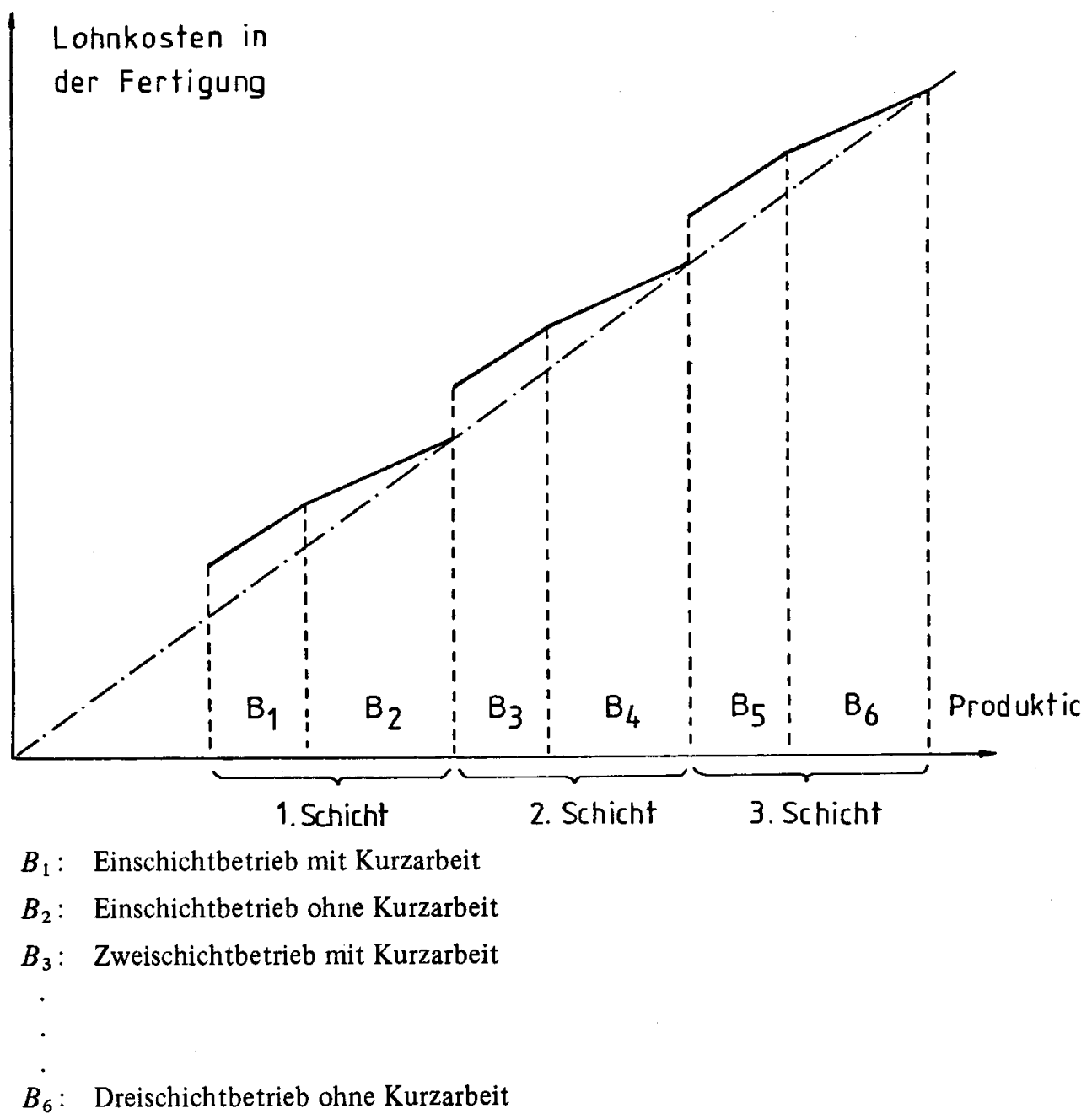

Abb. 2: Lohnkosten in der Fertigung

\section{Umstellungskosten}

Die Produktion ist ein äußerst komplexer Prozeß, der sich aus vielen verästelten und ineinander verwobenen Teilprozessen zusammensetzt. Dieser komplizierte Organismus besitzt einen optimalen Zustand. In ihm sind alle Einzelabläufe harmonisch aufeinander abgestimmt. Das ist der Punkt der kostengünstigsten Produktion. Führt man das Produktionssystem aus diesem optimalen Punkt über in einen neuen Optimalpunkt, weil sich die Nachfrage verschoben hat, dann dauert es eine gewisse Zeitspanne, bis sich das System in seiner neuen Lage eingeschwungen hat. In dieser Zeit entstehen erhöhte Kosten (Umstellungskosten, Kosten in der Materialbeschaffung usw.). Wenn man die Produktionsmenge dauern ändert, so operiert man stets im Bereich erhöhter 
Kosten. Günstiger ist es, dem System eine Chance zu geben, wenn es sich auf einem optimalen Zustand eingeschwungen hat, etliche Zeit in ihm zu verbleiben. Dann erst wird der Kostenvorteil wirksam. Die Veränderung der Produktionsrate soll also nicht stetig erfolgen, sondern schubweise mit genügend langen Pausen dazwischen. Man erreicht dies durch die Einfuhrung von fixen Umstellungskosten.

Stärker ins Gewicht fallen die Kosten bei Entlassungen und Einstellungen. Sie sind im wesentlichen proportional zur Veränderung des Personalbestandes.

\section{Aktionskosten}

Es ist eine typische Eigenheit des Kfz-Marktes, die Nachfrage durch Werbeaktionen in bestimmten Grenzen relativ zuverlässig beeinflussen zu können. Es werden dann Wagen mit einer festen Ausstattung verbilligt angeboten. Bei einem Nachlaß von a pro Stück werden zusätzlich $q$ (a) Stück abgesetzt.

\subsection{Optimierungsmodell}

Es ist das Ziel, die Produktionsrate entlang eines optimalen Kompromisses zwischen Lager-bzw. Fehlmengenkosten auf der einen Seite und niedrigen Nichtmaterialstiickkosten und Umstellungskosten auf der anderen Seite zu steuern. Als Optimierungskriterium verwenden wir die Bernoulli-Optimalität mit linearer Nutzenfunktion, d.h. wir minimieren den Erwartungswert der Gesamtkosten.

Als Prognose wird die Nachfrageschätzung (8) herangezogen. Das trifft die Situation aus Abschnitt 3.2, wo der Planungsabteilung die Langfristprognose bekannt ist.

\section{Notation:}

Für eine reelle Zahl $x$ sei definiert

$$
\begin{aligned}
& (x)^{+}= \begin{cases}x, & \text { falls } x>0 \\
0, & \text { sonst }\end{cases} \\
& (x)^{-}=(-x)^{+} \\
& \delta(x)= \begin{cases}1, & \text { falls } x \neq 0 \\
0, & \text { sonst. }\end{cases}
\end{aligned}
$$

Ferner seien $y^{-}, y^{+}$kritische Bestandsgrenzen

$$
\begin{aligned}
& y^{+} \text {Bestände über } y^{+} \text {werden in den Handel geschoben } \\
& y^{-} \text {Fehlbestände unter } y^{-} \text {verursachen Wartekosten }
\end{aligned}
$$


Die Gesamtkosten setzen sich zusammen aus den

Lagerkosten

$h_{1} y \quad$ (Vertriebslager)

$h_{2}\left(y-y^{+}\right)^{+} \quad$ (Händlerskonti)

\section{Fehlmengenkosten}

$g_{1} y \quad$ (Zins auf entgangenen Gewinn und Deckungsbeitrag für eine Periode)

$g_{2}\left(y^{-}-y\right)^{+} \quad$ (Ungeduld bei langen Lieferzeiten)

Material- und Energiekosten

$c\left(x_{t}+d_{t}\right)$

Nichtmaterialkosten

$F K_{t, i} \quad$ (Fixkosten im Bereich $B_{i}$ )

$P K_{t, i}\left(x_{t}+d_{t}-T P_{i-1}\right)$ (prop. Kosten im Bereich $B_{i}$ )

$x_{t}+d_{t} \in B_{i}$

\section{Umstellungskosten}

$k \cdot \delta\left(d_{t}\right)$

(fixe Umstellungskosten in der Feinsteuerung)

$e\left(x_{t}, d_{t}\right)$

Kosten bei Entlassungen)

$j\left(x_{t}, d_{t}\right)$

(prop. Kosten bei Einstellungen)

\section{Aktionskosten}

$a$

(Preisnachlaß pro Stück)

Minimierungsproblem

$\mathrm{Zu}$ minimieren ist die Zielfunktion

$$
E\left\{\left(\sum_{t=1}^{T} \text { Kosten in Periode } t\right) \mid\left(x_{1}, y_{1}\right)\right\} \rightarrow \begin{aligned}
& \min _{d_{1}, \ldots, d_{T}} \\
& a_{1}, \ldots, a_{T}
\end{aligned}
$$

Mit der Lagerbilanzgleichung als Nebenbedingung

$$
y_{t+1}=y_{t}+x_{t}+d_{t}-\hat{u}(t)-\epsilon-q\left(a_{t}\right)
$$

Die Zielfunktion läß̉t sich mit Hilfe eines instationären stochastischen Dynamischen Programms minimieren. Mit 
$L\left(x_{t}, y_{t}, d_{t}\right): \quad$ Erwartungswert der Lager- und Fehlmengenkosten im Zustand $\left(x_{t}, y_{t}\right)$ bei Produktion $x_{t}+d_{t}$

$v_{t}\left(x_{t}, y_{t}\right): \quad$ Erwartungswert der Gesamtkosten ab Periode $t$ bei Anfangszustand $x_{t}, y_{t}$

lautet das Dynamische Programm

$$
\begin{aligned}
v_{t}\left(x_{t}, y_{t}\right)= & \min _{d_{t}, a_{t}}\left\{k \cdot \delta\left(d_{t}\right)+e\left(x_{t}, d_{t}\right)+j\left(x_{t}, d_{t}\right)+c\left(x_{t}+d_{t}\right)+\right. \\
& +F K_{t, i}+P K_{t, i}\left(x_{t}+d_{t}-T P_{i-1}\right)+ \\
& +\beta \int v_{t+1}\left(x_{t}+d_{t}, y_{t}+x_{t}+d_{t}-\hat{u}(t)-\epsilon-\right. \\
& \left.\left.-q\left(a_{t}\right)\right) d P(\epsilon)\right\} \quad t=1,2, \ldots, T
\end{aligned}
$$

Die Planung erstreckt sich über einen Horizont $T$ von 12 Jahren. Was sich nach diesem Zeitpunkt abspielt, wird kostenmäßig in der Terminalbedingung ${ }{ }_{T+1}$ zusammengefaßt. Diese Kosten hängen von der Entwicklung des Prozesses jenseits des Planungshorizontes ab. Da diese unbekannt ist, läßt sich für die Festlegung von $v_{T+1}$ keine plausible Regel angeben. Jedoch beeinflußt der Zahlenwert von $v_{T+1}$ die Lösung des Optimierungsproblems. Damit ist die Terminalbedingung $v_{T+1}=V$ eine Fehlerquelle, die sich bei endlichem Planungshorizont prinzipiell nicht beseitigen läßt. Es entsteht ein Abbruchfehler, hervorgerufen durch den Abbruch der Antizipation zum Zeitpunkt $T$ infolge fehlender Daten.

Man verschafft sich Abhilfe, indem man den Planungshorizont um einige Perioden künstlich verlängert. Der Abbruchfehler entsteht dann erst später. Er pflanzt sich zwar rückwirkend fort, wird aber dabei stark gedämpft, denn auf die optimalen Entscheidungen in einer Periode $t$ üben zukünftige Ereignisse einen umso schwächeren Einfluß aus, je entfernter sie sind. Zahlreiche Beispielsrechnungen haben ergeben, daß eine Verlängerung um vier Perioden ausreicht.

\subsection{Ergebnisse}

\section{Analyse der Entscheidungsregeln}

Die Lösung der Funktionalgleichungen (11) liefert für jede Periode $t$ eine optimale Entscheidungsregel $\delta_{t}$ in Form einer Matrix $\left(d_{i j}^{*}\right)_{t}$ und einer Matrix $\left(a_{i j}^{*}\right)_{t}$. Die Entscheidungsregel besagt: Falls sich zu Beginn des $t$-ten Quartals das System im Zustand $\left(x_{t}, y_{t}\right)$ befindet, dann ändere die Produktionsrate um den Betrag $d_{i j, t}^{*}$ und starte (evtl.) eine Aktion mit dem Preisnachlaß $a_{i j, t} \geqslant 0$ ).

Da dem Verfasser keine Werbewirksamkeitskurve vorlag, wurde für die Berechnungen von der Möglichkeit einer Aktion kein Gebrauch gemacht, d.h. $a_{i j, t}=0$ f.a. $i, j, t$. 
Eine Analyse der Entscheidungssituation zeigt, daß sich das System plausibel verhält. Die optimalen Entscheidungsregeln spiegeln deutlich die Kostenstruktur wider. Die sprunghaften Kostenanstiege beim Übergang zu einer weiteren Schicht führen dazu, daß das System beim Übergang in den jeweiligen höheren Produktionsbereich träge ist. Es verharrt im alten Schichtbetrieb und verlangt erst dann eine zusätzliche Schicht, wenn diese genügend ausgelastet ist.

Kurzarbeit wird nur in den Perioden 3,6,7 und 10 in Erwägung gezogen und auch dann nur in geringem Umfang. Sie spielt insgesamt eine untergeordnete Rolle. Die Produktionsstückkosten im Kurzarbeitsbereich sind offenbar so, daß sich eine Personalkapazitätsvorhaltung nicht rentiert.

Wie man durch das Tal steuert

Im Innern der Produktionsbereiche ohne Kurzarbeit, mit Ausnahme des Einschichtbereiches, ist die Produktionsregel linear mit einem angesteuerten Lagerbestand am Ende der Periode von zwei Einheiten. Im Unterbeschäftigungsbereich steigt der angesteuerte Lagerendbestand auf drei Einheiten. Es läßt sich hieraus schließen, daß in der Regel die Mehrkosten im Unterbeschäftigungsbereich zu einer Produktionserhöhung um eine Einheit führen. Die erhöhten Lagerkosten werden durch ge-

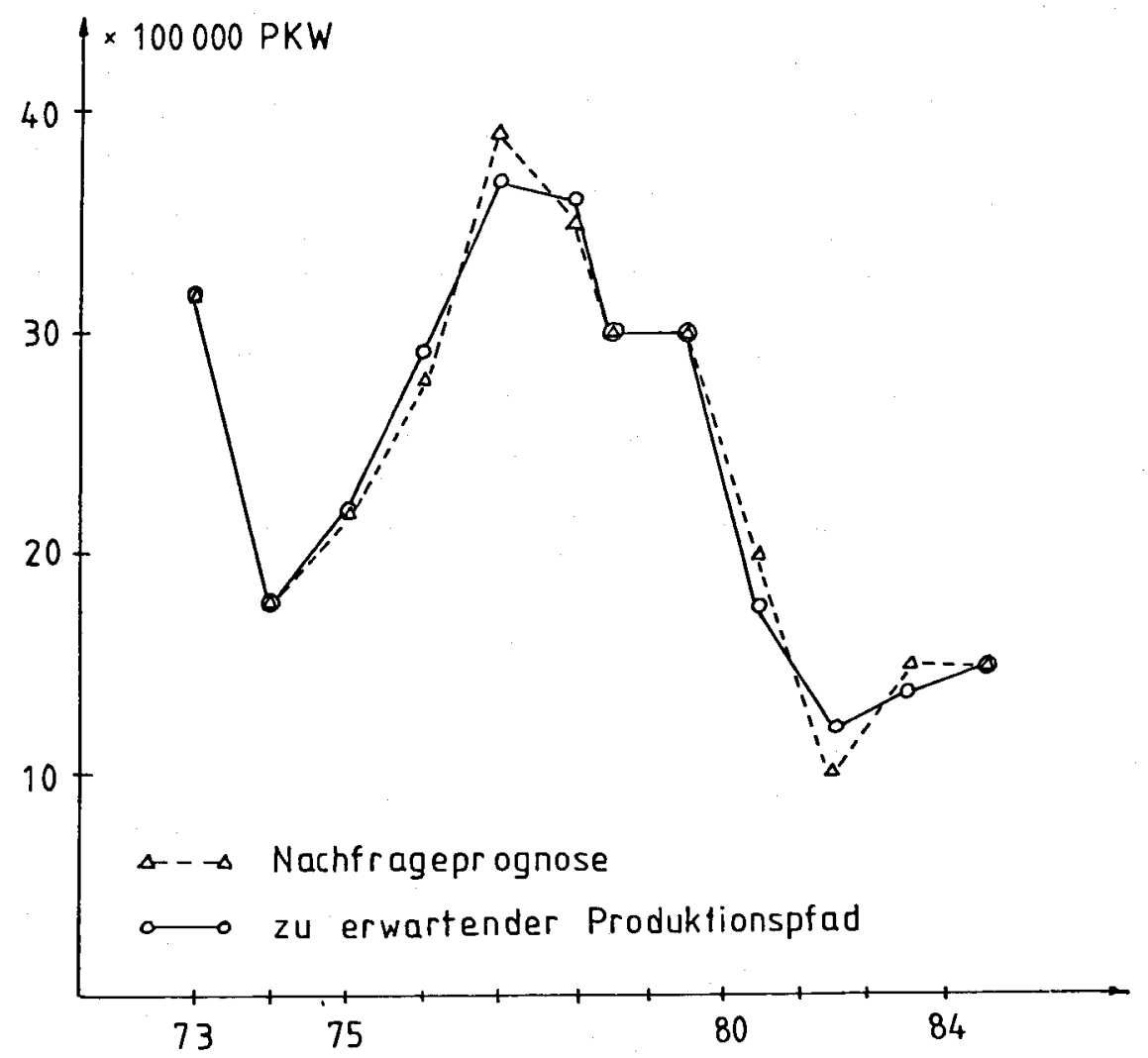

Abb. 3: Glättungswirkung des Modells 
ringere Stückkosten in der Herstellung kompensiert. Es besteht eine leichte Tendenz zur Überproduktion.

Man wird also zu Beginn der Talfahrt die Produktionsrate nicht ändern und das Lager um eine Einheit aufbauen. Was dann geschieht, hängt von der Entwicklung der Nachfrage ab. Um längerfristig planen zu können, kann man sich den wahrscheinlichsten Produktionspfad berechnen, wie er sich bei optimalem Handeln unter Risiko ergibt. Dann führt man eine Anzahl von Simulationsläufen unter Verwendung der Entscheidungsmatrizen $\left(d_{i j}^{*}\right)_{t}$ durch. Als wahrscheinlichsten Produktionspfad wählt man den Mittelwert aller simulierten Produktionspfade. Er ist in Abbildung 3 eingezeichnet. Danach ist zu erwarten, daß man die Produktionsrate auf siebzehn Einheiten absenkt (immer noch Zweischichtbetrieb), im zweiten Jahre im Überstundenbereich arbeitet und erst im dritten Jahr wieder um eine Schicht aufstockt.

\section{Vorteil gegenüber dem Modell mit festem Szenarium}

In der Abbildung 3 sieht man deutlich, wie die Nachfragespitze 1977 und der Nachfrageeinbruch 1982 in der Produktion weggeglättet werden. Dieser Glättungseffekt ist

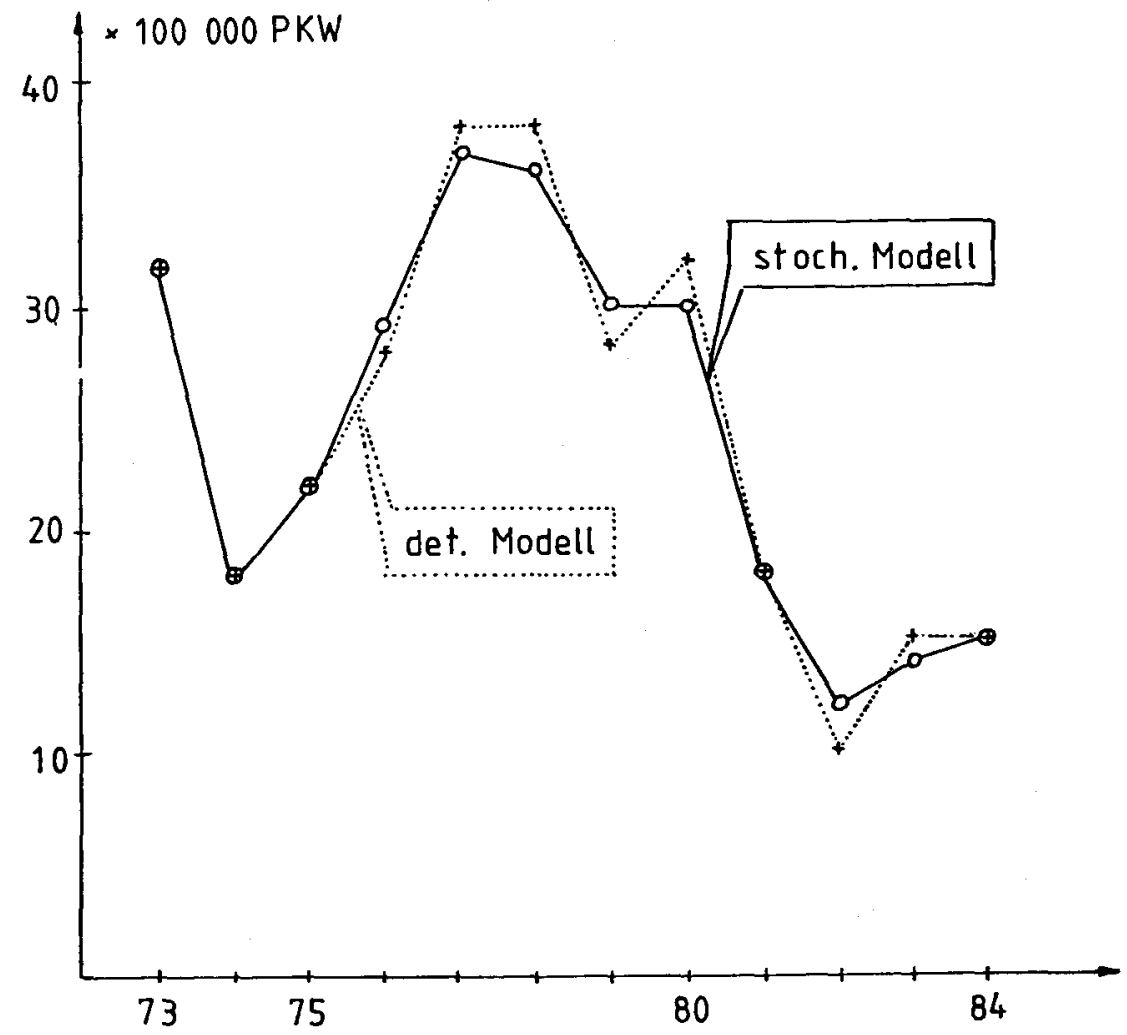

Abb. 4: Vergleich der zukünftigen Produktionsentwicklung aus dem stochastischen Modell mit unsicherem Szenarium (durchgezogen) und dem Modeli mit festen Szenarium (gestrichelt) 
zu erwarten. Er rührt daher, daß das Modell zu jedem Zeitpunkt die Zukunft vollständig bis hin zum Planungshorizont überblickt. Diese Antizipationsfähigkeit ist jedoch keine Eigenschaft, die allein dem stochastischen Modell (11) zuzuschreiben wäre. Auch ein deterministisches Modell antizipiert.

Es gibt jedoch neben der Antizipation noch einen weiteren Grund, der zur Glättung der Produktionskurve beiträgt, und dieser liegt ausschließlich im stochastischen Ansatz begründet. Befindet sich das System in einem ungünstigen Zustand (das ist ein Zustand, in dem selbst eine teuere Änderung immer noch billiger ist als gar keine Änderung), so behält das stochastische Modell stets die Chance im Auge, daß das System von selbst, also ohne eine mit Kosten verbundene Änderung, in einen günstigen Zustand übergeht. Die Wahrscheinlichkeit eines solchen Überganges kann u.U. hoch sein. In diesen Situationen wird das stochastische Modell noch keine Umstellung der Produktionsrate verlangen, das deterministische Modell hingegen schon. Der stochastische Ansatz verleiht dem Modell also eine zusätzliche Trägheitskomponente, die ,,Trägheit aus der Wahrscheinlichkeit".

Wie groß dieser stochastische Glättungseffekt ist, läßt sich aus der Gegenüberstellung in Abbildung 4 erkennen. Dort ist neben dem wahrscheinlichsten Produktionspfad aus dem stochastischen Modell der Produktionspfad aus dem Modell mit festem Szenario (gestrichelte Linie) aufgezeichnet.

Es ist zu erkennen, daß der Glättungseffekt, hervorgerufen durch die Risikokalkulation des stochastischen Modells, beträchtlich ist. Dieses Ergebnis bestätigt die qualitative Uberlegenheit der stochastischen Langfristplanung bei unsicherem Szenario gegenüber dem Modell mit festem Szenario. Allein unter dem Gesichtspunkt der Kostenminimierung liefert das stochastische Modell eine glattere und damit auch sozialverträglichere Produktionssteuerung.

\section{Literaturverzeichnis}

Bartmann, D.: Stochastische Produktionsglättungsmodelle bei stationärer und nichtstationärer Nachfrage. In: Proceedings in Operations Research 8. Hrsg. v. K.-W. Gaede. Heidelberg 1979, 354-360.

-: Optimale Produktionsplanung bei Frischprodukten mit dem Programm FORTUNA - eine Fallstudie. Zeitschrift für Operations Research 27, 1983, B179-B204.

-: Reduction of State Space in Dynamic Programming with Integrated Forecasting. In: Operations Research and Economic Theory. Eassay in Honour of Martin J. Beckmann, Hrsg. v. Hauptmann, Krelle, Mosler. Heidelberg 1984.

Beckmann, M.J.: Production Smoothing and Inventory Control. Operations Research 9, 1961, $456-467$.

Eppen, G.D., und E.F. Fama: Cash Balance and Simple Dynamic Portfolio Problems with Proportional Costs. Int. Econ. Revue 10, 1969, 119 133.

Hochstädter, D.: Stochastische Lagerhaltungsmodelle. Lecture Notes in Economics and Mathematical Systems 10. Heidelberg 1974.

Inderfurt, $K$.: Optimale Kassenhaltung bei beschränktem Geldtransfer und Kassenbestand. Zeitschrift für Betriebswirtschaft 48, 1978, 651-665.

Schneeweiß, Ch.: Inventory-Production Control. Lecture Notes in Economics and Mathematical Systems 151. Heidelberg 1977.

Streißler, E. (Hrsg.): Information in der Wirtschaft. Schriften des Vereins für Socialpolitik, N.F., 126, Berlin-München 1983. 